\title{
In Fiber Resonance Breaking Mechanism
}

\author{
Osman Akın \\ Electrical\&Electronics Engineering \\ Izmir Institute of Technology \\ Izmir, Turkey \\ osmanakin@iyte.edu.tr
}

\author{
M. Salih Dinleyici \\ Electrical\&Electronics Engineering \\ Izmir Institute of Technology \\ Izmir, Turkey \\ salihdinleyici@iyte.edu.tr
}

\begin{abstract}
In this work we investigate mode resonance breaking mechanism in slab waveguide accomplished by cladding index modulation according to Kerr nonlinearity. The interaction is considered to be a Four Wave Mixing Process and Coupled Mode Theory is used to analyze the effect of cladding perturbation into propagating fundamental mode.
\end{abstract}

Keywords - Third order optical nonlinearity; resonance breaking; Four Wave Mixing;Transient grating;

\section{INTRODUCTION}

A mode resonance breaking is an important phenomenon that can play a fundamental role in all optical signal processing applications and especially in ultrafast all optical switching elements. Realizing processes in all optical manners involve controlling of light with light that requires highly nonlinear medium with instantaneous response time. With the development of polymer science, materials with high third order nonlinearity, namely Kerr nonlinearity having optical susceptibility of about $10^{-7}$ esu was synthesized [1]. Furthermore, a response time of femtoseconds for the Kerr type materials was reported [2], which makes Kerr type materials a suitable medium for such applications.

The transverse resonance breaking can be achieved by altering the cladding refractive index of the waveguide. $\mathrm{N}$. Chen et al. discovered that a periodic perturbation created in cladding can affect the fundamental core mode [3]. Ma and Wolff analyzed guiding characteristics of a waveguide having nonlinear substrate [4].

In this study we investigate the dynamics of resonance breaking mechanism with nonlinear refractive index of a transient grating created in the core-cladding boundary of the waveguide as shown in figure 1.

The structure is created by partial removal of the cladding and placing a material with high third order nonlinearity instead of a cladding material. The Kerr type nonlinear medium enables the interaction of propagating fundamental mode with laser control light forming grating that breaks the resonance of the mode. The proposed waveguide structure can be analyzed by considering Four Wave Mixing (FWM) of Gaussian beams and propagating mode in the region of core-cladding boundary

This work has been supported by Tubitak, Project No: 109E240

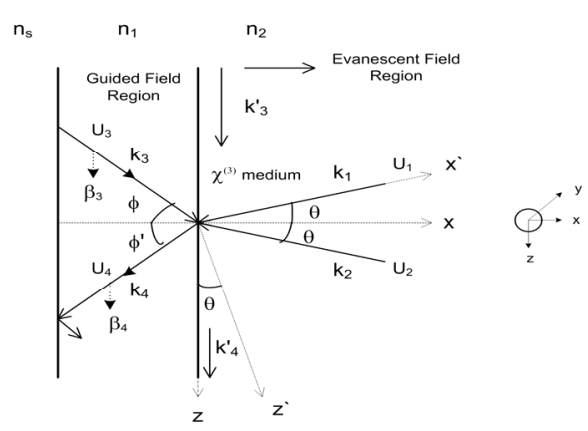

Figure 1. Four wave mixing at the core cladding boundary

where fields of modes and grating forming fields are interacting in the evanescent region of the waveguide and where nonlinear material is placed. Hereafter, the coupled wave equations for the propagating modes are obtained in the existence of the transient grating.

\section{RESONANCE BREAKING WITH TRANSIENT GRATING}

A slab waveguide with a cladding composed of third-order nonlinear material is considered. The fundamental mode propagating in the waveguide is represented by $U_{3}$ with propagation constant $\beta_{3}$ and higher order mode $U_{4}$ with propagation constant $\beta_{4}$ is generated after the breaking of the waveguide resonance due to the effect of control light. The interaction of the propagating mode with the generated transient grating can be considered as a Four Wave Mixing (FWM) process in which grating forming beams $U_{1}, U_{2}$ and the propagating waveguide mode $U_{3}$ interact with each other and a fourth wave $U_{4}$ is formed as a result of this FWM interaction. In this resonance breaking mechanism fundamental mode $U_{3}$ continues to propagate but loose its power for the benefit of the higher order mode $U_{4}$.

The grating forming Gaussian beams $U_{1}$ and $U_{2}$ at wavelength $\lambda_{L}$ cross at half angle $\theta$ at the cladding side of the core-cladding boundary and create pattern that modifies 
cladding refractive index. Assuming that both grating writing beams are originating from the same source; their wavelengths, beam magnitudes and waists can be considered equal. Although the interference equation consists of traveling and standing waves, a grating pattern is created by the stationary wave given as [5]:

$$
\begin{array}{r}
\Psi(x, y, z)=\frac{A^{2}}{|\rho|^{2}} \exp \left(-k_{L} x_{0} \frac{y^{2}+(x \sin (\theta))^{2}+z^{2}}{|\rho|^{2}}\right) \\
\cdot \cos \left[2 k_{L} \sin \theta\left(1-x \frac{x-L}{|\rho|^{2}}\right) z\right]
\end{array}
$$

where $|\rho|^{2}=\left|x^{\prime \prime}-L\right|^{2}+\left|x_{0}\right|^{2}, k_{L}=\frac{2 \pi}{\lambda_{L}}$ and $x^{\prime \prime}$ the intersection plane $x=x^{\prime \prime}$. The exponential part of this equation defines a Gaussian profile for the grating. The coefficient $k_{L} \frac{x_{0}}{|q|^{2}}$ acts like a diminishing factor in three dimensions, while grating period in the $\mathrm{z}$ direction depends on the intersection angle $\theta$, writing beam wavelength $\lambda_{L}$

The total field in the interaction region can be written as the superposition of the ideal modes, as follows

$U_{3}=\sum_{m} A_{m}(z) E_{m}(x, y) \exp \left\{-j \beta_{m} z\right\}$

$U_{4}=\sum_{n} B_{n}(z) E_{n}(x, y) \exp \left\{-j \beta_{n} z\right\}$

Where $\mathrm{A}_{\mathrm{m}}$ and $\mathrm{B}_{\mathrm{n}}$ are slowly varying amplitudes, $E_{m}$ is the transverse field distribution of incident (fundamental) mode (m) and $E_{n}$ is the transverse field distributions of the higher order mode (n).

By using FWM matching conditions $\vec{k}_{1}+\vec{k}_{3}=\vec{k}_{2}+\vec{k}_{4}$ and $\omega_{1}+\omega_{3}=\omega_{2}+\omega_{4}$, the Helmholtz equations for incident and outgoing waves can be written as [6]:

$$
\begin{aligned}
& \left(\nabla^{2}+k_{3}^{2}\right) U_{3}=-\zeta_{3} \Psi U_{4}-\gamma_{3} U_{3} \\
& \left(\nabla^{2}+k_{4}^{2}\right) U_{4}=-\zeta_{4} \Psi^{*} U_{3}-\gamma_{4} U_{4}
\end{aligned}
$$

where $\zeta_{3,4}=6 \mu_{0} \omega_{3,4}^{2} \chi^{(3)}, \gamma_{3,4}=2 n_{c l} n_{2} \frac{\omega_{3,4}^{2}}{c_{o}^{2}}\left(2 I-I_{3,4}\right), n_{c l}$ is the cladding refractive index, $n_{2}$ is the Kerr coefficient, $I=I_{1}+I_{2}+I_{3}+I_{4}$ is the total intensity and $I_{3,4}=\frac{\left|U_{3,4}\right|^{2}}{2 \eta_{0}}, \chi^{(3)}$ is the third order nonlinear susceptibility and $\Psi=U_{1}^{*} U_{2}$ where $U_{1}$ and $U_{2}$ are the grating forming fields.

When we substitute (2) and (3) into (4) and (5), we obtain coupled mode equations for every mode:

$$
\frac{d A_{m}}{d z}=-j \mathrm{~K}_{m}(z) B_{n}(z) \exp \left\{-j\left(\beta_{n}-\beta_{m}\right) z\right\}+j\left(-\frac{\gamma_{3}}{2 \beta_{m}}\right) A_{m}(z)
$$

$$
\frac{d B_{n}}{d z}=-j \mathrm{~K}_{n}(z) A_{m}(z) \exp \left\{j\left(\beta_{n}-\beta_{m}\right) z\right\}+j\left(-\frac{\gamma_{4}}{2 \beta_{n}}\right) B_{n}(z)
$$

Where $\mathrm{K}_{m}$ and $K_{n}$ are mode coupling coefficients denoted as:

$\mathrm{K}_{m}(z)=\frac{\left|\beta_{m}\right|}{\beta_{m}} \frac{1}{4 \omega \mu} \zeta_{3} C_{m n}(z)$

$\mathrm{K}_{n}(z)=\frac{\left|\beta_{n}\right|}{\beta_{n}} \frac{1}{4 \omega \mu} \zeta_{4} C_{n m}(z)$

and $C_{n m}$ is the overlap integral coefficient, which can be written as:

$C_{n m}(z)=\iint_{S} \Psi(x, y, z) E_{m}(x, y) E_{n}^{*}(x, y) d x d y$

where $\Psi(x, y, z)$ is the corrugation (grating) function that disrupts the orthogonality of transverse fields.

The exact solution for (6) and (7) is possible only for uniform grating structure. Because the grating function $\Psi$ exhibits non-uniform behavior, Fourier series analysis can be used to find the fundamental mode amplitude $\mathrm{A}$ and higher order mode B [7]. Applying boundary conditions $A(0)=A_{0, p, r}$, $B(0)=0$ and assuming that total optical power is conserved within the waveguide $\left[\frac{d}{d z}\left(\left|A_{m}\right|^{2}+\left|B_{n}\right|^{2}\right)=0\right]$, the solution for 
each Fourier harmonic of the fundamental mode can be written as:

$$
\begin{aligned}
A_{p, r}(z)= & {\left[A_{0, p, r} \cos \left(Q_{p, r} z\right)+j \frac{A_{0, p, r}}{Q_{p, r}} \widehat{\delta}_{p, r} \sin \left(Q_{p, r} z\right)\right] } \\
& \cdot \exp \left(-j \delta_{p, r} z+j \frac{\alpha_{a}+\alpha_{b}}{2} z\right)
\end{aligned}
$$

Considering the solution of the coupled equations (6) and (7) for each harmonic q corresponding to each Fourier coefficient parameter ( $\mathrm{p}, \mathrm{r})$ related to the grating function (1), total amplitude of the propagating fundamental mode in the waveguide can be written as:

$$
A_{m}(z)=\sum_{q} A_{q}(z)=\sum_{p, r} A_{p, r}(z)
$$

We find the $Q$ parameter as $Q_{p, r}^{2}=\mathrm{K}_{p, r}^{2}+\widehat{\delta}_{p, r}^{2}$, where the detuning parameter $\widehat{\delta}_{p, r}$ is written in terms of the phase matching parameter $\delta_{p, r}$ and self coupling coefficients $\alpha_{a}$ and $\alpha_{\mathrm{b}} \quad$ as $\quad \hat{\delta}_{p, r}=\delta_{p, r}+\frac{\alpha_{a}-\alpha_{b}}{2}$. Self coupling coefficients arise from the third order nonlinearity and depend on the Kerr nonlinearity and total intensity I. These coefficients can be written as: $\alpha_{a, b}=-\frac{2 n_{c l} n_{2} \frac{\omega_{3,4}^{2}}{c_{0}^{2}}\left(2 I-I_{3,4}\right)}{2 \beta_{3,4}}$

\section{RESULTS}

The grating profile formed by the interference of Gaussian beams in the cladding of the waveguide is illustrated in Fig. 3. Assume that cladding is made of polymeric nonlinear material with refractive index $n_{c l}=2.32$ and the core refractive index is $n_{\text {core }}=2.4$. This grating is formed by laser beams of wavelength $532 \mathrm{~nm}$ and an intersection half angle of $\theta=0.6^{\circ}$. The beam minimum waist is $W_{0}=1 \mathrm{~mm}$ and the distance between laser minimum waist location and intersection point is $L=1 \mathrm{~mm}$. The period of the resulting grating is $\Lambda=25.4 \mu \mathrm{m}$.

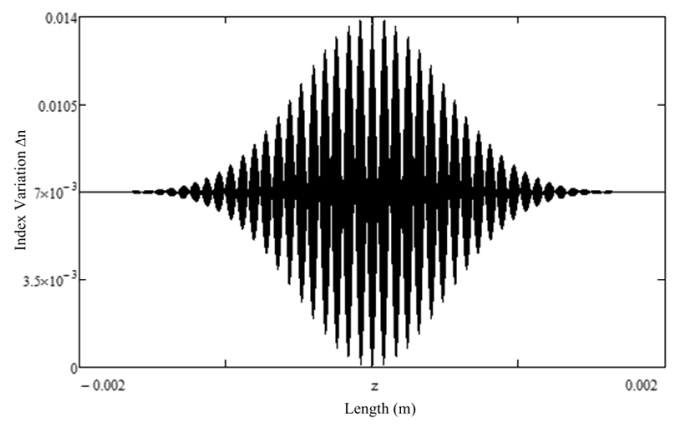

(a)

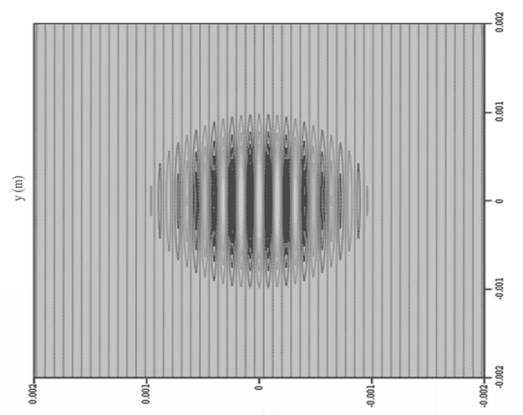

(b)

Figure 2. a-) 1D Intensity-Dependent Refractive Index pattern b-) Contour plot of interference fringes

When no index variation is present in the cladding, all of the power is concentrated in the fundamental mode. The formation of index modulation at the cladding region breaks the resonance of the waveguide and yields power dissipation in fundamental mode providing energy transfer into the higher order mode. The power loss along grating is shown in figure 3.

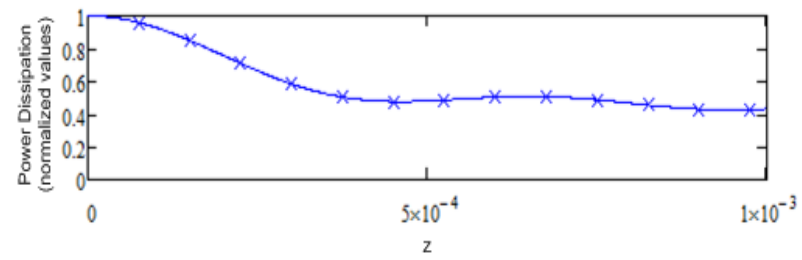

Figure 3. Power loss in fundamental mode through grating

On the other hand, the fraction of power loss depends on the maximum value of index variation. For $\Delta n=0.001$, nearly $82 \%$ of the total power remains in fundamental mode at the end of the grating. In contrary, when the index variation is increased to $\Delta n=0.009$, fundamental mode looses $60 \%$ of its energy just at the beginning of the transient grating as shown in figure 4

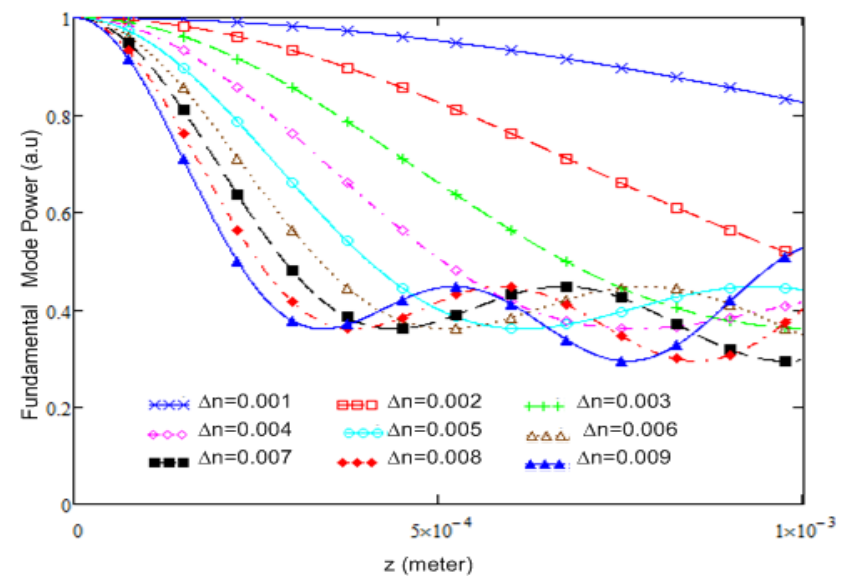

Figure 4. The change in fundamental mode power according to the maximum refractive index change $\Delta n$ 


\section{CONCLUSION}

A resonance breaking mechanism in a slab waveguide was analyzed by using coupled mode theory. This structure depends on the refractive index change in the cladding according to the Kerr effect introduced by externally applied Gaussian beams. Simulation results imply that the modification of the cladding index causes fundamental mode to loose its energy. For cladding index variation of $\Delta n=0.009$, fundamental mode loose nearly $\% 60$ of its energy. By taking advantages of fast response time of Kerr type materials, it is possible to implement this phenomenon in high speed all optical switching applications with low control light requirements.

\section{REFERENCES}

[1] X. Zhan, Y. Liu, D. Zhu, X. Liu, G. Xu, P. Ye, "Large third-order nonlinear optical response of a conjugated copolymer consisting of 2,5diethynylthiophene and carbazole units," Chemical Physics Letters, Vol. 343, issue 5-6, pp. 493-498, Aug. 2001.

[2] T. Schneider, D.Wolfframm, R.Mitzner, J.Reif, "Ultrafast optical switching by instantaneous laser-induced grating formation and selfdiffraction in barium fluoride," Applied Physics B Lasers and Optics, vol. 68, issue 4, pp. 749-751, Apr. 1999.

[3] N. Chen, B. Yun, Y. Cui, "Cladding index modulated fiber grating," Optics Communications, vol. 259, 2006.

[4] G.J. Ma and I. Wolff, "Propagation characteristics of TE waves guided by thin films bounded by nonlinear media," IEEE Trans MTT, vol. 43, 1995.

[5] O. Akin, "All Optical Switching Via Diffraction Grating Formed By Interference of Gaussian Beams," MSc Thesis, Izmir Institute of Technology, 2005.

[6] B.E.A. Saleh and M.C. Teich, Fundamentals of photonics. John Wiley \& Sons, 2007.

[7] O. Akin, M.S. Dinleyici, "An All-Optical Switching Based on Resonance Breaking with a Transient Grating", submitted to IEEE Journal of Lightwave Technology 